MATERNAL MORTALITY IN AUSTRALIA: WHAT CAN WE LEARN FROM STORIES OF POSTPARTUM HAEMORRHAGE?

Caroline Homer RM PhD

Professor of Midwifery

University of Technology Sydney

Vanessa Clements RM

Area Clinical Midwifery Consultant

Midwifery Practice Development \& Research

South Eastern Sydney Illawarra Area Health Service

Nolan McDonnell BHB MBChB FANZCA

Staff Specialist

Department of Anaesthesia and Pain Medicine

King Edward Memorial Hospital for Women

Western Australia

Michael Peek

Professor of Obstetrics and Gynaecology

Associate Dean and Head

Nepean Clinical School

University of Sydney

Nepean Hospital, NSW

Elizabeth Sullivan

Associate Professor and Director

Perinatal and Reproductive Epidemiology Research Unit

(Incorporating AIHW National Perinatal Statistics Unit)

School of Women's and Children's Health

University of New South Wales 


\title{
MATERNAL MORTALITY IN AUSTRALIA: WHAT CAN WE LEARN FROM STORIES OF POSTPARTUM HAEMORRHAGE?
}

\begin{abstract}
Death from pregnancy is rare in developed countries such as Australia but is still common in third world and developing countries. A significant disparity exists in maternal mortality in Australia. The maternal mortality ratio for Indigenous women remains over three times that for other Australian women over the nine year period 1997- 2006. The investigation of each maternal death yields valuable information and lessons that all health care providers involved with the care of women can learn from. The ultimate aim of these investigations is to prevent future maternal morbidity and mortality.
\end{abstract}

Obstetric haemorrhage remains a leading cause of maternal death internationally. It is the most common cause of death in developing countries. In the latest reports from Australia and the UK obstetric haemorrhage is ranked as the $4^{\text {th }}$ and $3^{\text {rd }}$ most common cause of direct maternal death respectively. In a number of cases there are readily identifiable factors associated with the care that the women received that may have contributed to their death. It is from these identifiable factors that both midwives and doctors can learn to help prevent similar episodes from occurring.

This article is the first in a series that will identify some of the lessons that can be learnt from the recent Australian and UK maternal death reports. This paper presents an overview of the process and systems for the reporting of maternal death in Australia. It will then specifically focus on obstetric haemorrhage, with a focus on postpartum haemorrhage, for the 12 year period, 1994-2005. Vignettes from the maternal mortality reports in Australian and the United Kingdom are used to highlight the important lessons for providers of maternity care.

Word count: 4500wds 


\section{Introduction}

Maternal death in developed countries such as Australia and the United Kingdom (UK) is rare. In these countries, pregnancy and childbirth is, for the most part, a normal and happy event culminating in the birth of the baby and a healthy mother. Maternal death is not only unexpected, but a tragedy for all involved. There are often valuable lessons that can be learnt. These lessons have the potential to prevent future deaths and significant maternal morbidity occurring under similar circumstances. It is important that all health care providers caring for pregnant women learn from these experiences.

Maternal death is defined by the World Health Organization (WHO) as a death occurring anytime during pregnancy and up to 42 days postpartum. A late maternal death is one that occurs between 42 days and one year after pregnancy [1]. Maternal death is used as a benchmark of the country's overall health and development status. A key goal of the United Nations Millennium Project (which runs from 1990 to 2015) is to reduce the maternal mortality ratio by three quarters [2].

Maternal death is further classified into direct, indirect and incidental deaths. Direct deaths result from obstetric complications (eg, obstetric haemorrhage, eclampsia). Indirect deaths result from an illness or condition that was in some way exacerbated by the pregnant state (eg, cardiac disease, psychiatric illness). Incidental deaths are those unrelated to the pregnancy (eg. road traffic accidents) $(1,3)$.

Many countries report on their maternal deaths triennially (every three years) in order to make the data meaningful and to allow for recommendations to be made. It has long been recognised that maternal deaths are just the tip of the "iceberg". The WHO report "Beyond the Numbers" makes comment that "for every woman who dies, many more suffer from serious conditions that can affect them for the rest of their lives" (4).

In contrast to other countries, it is not mandatory to report a maternal death in Australia (5). In recent years, a maternal death in hospital is reported nationally as a Sentinel Event (5). Maternal deaths are notified to individual state or territory Health Department Maternal Mortality Committees via a number of different avenues. These include: individual practitioner reporting; coronial investigations and the register of Births, Deaths and Marriages. Each committee is made up of a multidisciplinary team including midwives; obstetricians; anaesthetists; pathologists; physicians; perinatal psychiatrists and epidemiologists. A confidential enquiry is conducted on each death, the aim is to identify a principal cause of the death, assign a classification as well as to identify any potentially avoidable factors. Enquiry findings are forwarded to the National Advisory Committee on Maternal Mortality who review all deaths and collate a report every three years (5). The triennial report is produced under the auspices of the Australian Institute of Health and Welfare (AIHW) with the most recent report funded by the Australian Commission on Safety and Quality in Health Care. There are significant limitations with the current system as not all states and territories have active maternal mortality review committees and it is likely that not all maternal deaths are accounted for in Australia (5).

Australia (6) and the UK (7) have recently published their most recent reports. These reports contain useful and important lessons for all health professionals involved in 
the care of childbearing women. These lessons have relevance for midwives and others working in maternity care. This article is the first in a series that will identify some of the lessons that can be learnt from the Australian and UK maternal death reports.

Globally, obstetric haemorrhage remains the most significant cause of maternal mortality (8). It is estimated that postpartum haemorrhage is the most common cause of maternal death across the world, responsible for more than $25 \%$ of deaths annually (9). Obstetric haemorrhage is also a significant cause of severe maternal morbidity. A recent Scottish study identified major haemorrhage as accounting for $69 \%$ of the cases of severe maternal morbidity and $11 \%$ of direct maternal deaths (4). For this reason, obstetric haemorrhage is the topic of this paper.

This article provides an overview of the most recent report on maternal deaths in Australia for the triennia 2003-2005. It then focuses on obstetric haemorrhage, with a focus on postpartum haemorrhage, making reference to the data for the 12 year period, 1994-2005. Short vignettes or stories of real cases from the Australian and UK maternal mortality reports are used to highlight a number of the issues and learning points for midwives and maternity services. Future articles will address amniotic fluid embolism, hypertension, thrombosis and thromboembolism and cardiac disease. We hope this series of articles will encourage midwives and other clinicians to read the reports from which these stories come, to reflect on the findings and recommendations and also how they might impact on their own practice.

\section{Approach}

Data were sourced from published reports of maternal deaths in Australia and the United Kingdom (UK). These are observational data and the numbers presented need to be interpreted with caution due to the small numbers, and variability in ascertainment by time and the different definitions of viability in the two countries. The maternal mortality ratio is used to report maternal mortality. It is defined for international use as the number of maternal deaths (direct and indirect) over a given period per 100000 live births over the same period.

The National Perinatal Statics Unit in Australia and the Confidential Enquiry in Maternal and Child Health (CEMACH) in the UK provided permission to use the vignettes that have been selected to illustrate the best practice principles. The source of these vignettes is presented under each of the Figures.

\section{Overview of maternal deaths in Australia 2003-2005}

There were 65 maternal deaths in the triennium 2003-2005. Selected characteristics of these deaths are summarised in Table 1 . There has been a decline in the MMR from 12.8 per 100,000 women giving birth in 1976-1978 to 8.4 per 100,000 women giving birth in 2003-2005. This is largely due to a decline in direct deaths over the period. The data was however not validated against other databases (eg Australian Bureau of Statistics mortality data and National Hospital Morbidity Database) so it is possible that the true number of deaths is higher than reported. 
The majority (72\%) of maternal deaths reported in 2003-2005 deaths underwent a post mortem examination but this varied by classification. The MMR for Australian Indigenous women was 2.9 times that of non-Indigenous women. Younger and older women had a higher MMR compared with women aged 20-34 years. The most common cause of direct maternal death was amniotic fluid embolism (28\%). The other causes included hypertensive disorders (17\%); thrombosis and thromboembolism (17\%); and, obstetric haemorrhage (14\%). Almost half (41\%) of deaths were in women aged less than 30 years and half (52\%) of the women were nulliparous or primiparous. The most common cause of indirect deaths was attributed to the category of cardiac conditions (28\%). The other causes included psychiatric conditions (17\%), non-obstetric haemorrhage (14\%) and infection (11\%) (Table 1).

$<$ Table 1 here $>$

\section{Obstetric haemorrhage}

In Australia and the UK between 1994 and 2005 (10-15) there were seventy-four deaths directly attributed to obstetric or uteroplacental haemorrhage (Table 1). Obstetric or uteroplacental haemorrhage accounted for 10 to $17 \%$ of all direct deaths in Australia and the UK in this time period.

<Table 2 here $>$

Recent reports from Australia and Canada confirm the increasing incidence of postpartum haemorrhage (16-19). In Australia, it has been suggested that this increase is related to changes in management and/or reporting of postpartum haemorrhage (18). In Canada, the reason for the increase appears to be related to the rates of atonic postpartum haemorrhage, the causes of which are unknown and do not seem to be affected by either maternal age or previous caesarean section (16). Postpartum haemorrhage may occur for the first time in any pregnancy, and may recur in subsequent pregnancies (17).

Haemorrhage in postpartum women can be massive and abrupt in onset. The blood flow to the uterus at term is between $7-800 \mathrm{mls} / \mathrm{min}$ and the uterus receives $20-25 \%$ of the bodies total cardiac output. The haemodilution and increased blood volume that occurs in pregnancy are likely to have evolved as a protective mechanism against blood loss associated with delivery. As past of the normal physiological changes of pregnancy and delivery the heart rate increases and the blood pressure can decrease, these changes can mask the early subtle signs of a severe postpartum haemorrhage. Prompt, aggressive and expert care is required as the potential for rapid ongoing blood loss is high. All maternity care clinicians should be familiar with the management of postpartum haemorrhage, have a clear plan in place for its management and be prepared to call for urgent help earlier rather than later.

\section{Vignettes}

Four vignettes of women whose deaths were attributed to obstetric haemorrhage have been selected from the Australian and UK reports. These cases broadly identify many of the themes and highlight some of the lessons that midwives can learn from maternal mortality reports. 


\section{Care of a woman who refused blood products}

A 22-year-old woman having her first baby was admitted to hospital following an uneventful pregnancy for a post-dates induction of labour which resulted in a normal vaginal birth of a liveborn infant. She had an immediate postpartum haemorrhage of approximately $600 \mathrm{ml}$ and was treated with intravenous (IV) Syntocinon and Hartmanns solution. Approximately four hours later she suffered another postpartum haemorrhage of approximately $400 \mathrm{ml}$, which was treated with a further Syntocinon infusion. The placenta was documented to be complete. Over the next two days she had moderate lochia rubra loss and reported feeling dizzy. On postpartum day 3 she experienced a third postpartum haemorrhage of $500 \mathrm{ml}$ and complained of feeling faint and cold. She was treated with IV fluids, bed rest and Haemaccel. The woman signed a medical release declining blood transfusion under any circumstances. An IV Syntocinon infusion was titrated to blood loss. She continued to have moderate loss of lochia rubra. On day four, with haemoglobin value $55 \mathrm{~g} / \mathrm{L}$, the intravenous catheter was removed. On day five, with haemoglobin $59 \mathrm{~g} / \mathrm{L}$, she was discharged home on iron tablets. She was re-admitted to hospital on day 11 following a further postpartum haemorrhage of $500 \mathrm{ml}$. Cervical dilatation and uterine curettage were carried out and placental tissue was removed. The woman was kept cool, intubated, sedated and paralysed after the procedure to reduce oxygen requirements and maintained on Haemaccel, inotropic support and IV fluids. She was transferred for hyperbaric oxygen treatment. She received three treatments, but rapidly deteriorated over the next two days and developed cardiac failure secondary to gross anaemia and died on day 13 postpartum. A postmortem examination was not carried out and the cause of death was deemed to be cardiac failure due to severe anaemia secondary to postpartum haemorrhage.

Source: Reproduced from Report on maternal deaths in Australia 1994-96. Ford et al. Chapter 9: Uteroplacental haemorrhage, page 59, 2001, with the permission of the National Perinatal Statistics Unit (12).

This vignette outlines a slow deterioration with progressive intermittent bleeding over a number of days. None of the bleeding episodes were large in isolation however, over time and collectively, these repeated episodes of bleeding were significant. This vignette highlights the need for vigilance and ongoing monitoring and intervention, especially after the immediate postpartum hours. The diagnosis of retained products rather than uterine atony perhaps should have been entertained earlier.

The other complicating factor was the woman's decision to refuse blood or blood products. Of particular concern are the challenges that it raises for midwives, medical staff and the multidisciplinary team as often these women would not have died if they had received blood. The 2000-2002 CEMACH Report described the distress for staff involved in such cases, especially if the woman subsequently dies, and recommended that additional support should be made available [14]. Clinicians have a role in supporting the woman in her choices even though they may be at odds with their own beliefs. The families of these women will also require the same support as any other bereaved family.

A number of lessons can be highlighted from this vignette. While this is a story from the 1994-1996 report, many of the issues remain current and applicable today. Guidelines should be in place regarding the management of women who refuse blood products [3]. This management plan should include: booking for birth in a unit that 
has facilities for prompt management of haemorrhage and ICU; careful measurement of haemoglobin and prevention of anaemia in the antenatal period; identification of the placental site by ultrasound in late pregnancy; vaginal birth if possible rather than caesarean section as the blood loss is generally less; consultation and referral to a consultant obstetrician and anaesthetist through pregnancy and when the woman is admitted in labour; and, active management of the third stage of labour. If haemorrhage does occur, blood loss must be carefully measured and clotting status investigated promptly. Interventions such as embolisation of the uterine arteries, BLynch suture and intravenous vitamin K may be necessary and should be undertaken sooner rather than later. The use of intra operative cell salvage is becoming more popular in major obstetric haemorrhage and it is acceptable to most Jehovah's Witnesses. Finally, support should be available for staff following such an event.

\section{A woman with acute blood loss leading to collapse}

A 34 year old woman having her second baby developed hypertension at about 32 weeks gestation. She presented to labour ward at 38 weeks gestation feeling unwell, was induced and proceeded to normal vaginal birth nine hours later. Her uterus became atonic about one hour after delivery with 1,000 $\mathrm{ml}$ of blood clot expressed from the uterus. Six hours post the birth she had a second 1,000 $\mathrm{ml}$ of blood clot expressed from the uterus. An examination under anaesthesia was carried out. There was no evidence of trauma found, no retained products, the uterus was 'somewhat' atonic. She developed acute severe pulmonary oedema in the recovery room and had a cardio-respiratory arrest about two hours later. Attempts to resuscitate her for several hours were unsuccessful.

Source: Reproduced from Maternal deaths in Australia 1997-1999. Slaytor EK, Sullivan EA \& King J. Chapter 3: Obstetric haemorrhage, page 18, 2004, with the permission of the National Perinatal Statistics Unit (13).

This vignette has many similarities to the first and highlights the risks of an atonic uterus. The immediate management of the first episode of bleeding is unclear in the report [12] however, one could question its adequacy due to the recurrence of bleeding. Resuscitation of pregnant women can be more complex because of the physiological changes of pregnancy. These changes can predispose to the development of pulmonary oedema which is also a rare but well document complication of blood transfusion. This vignette also highlights the rapid deterioration that can occur in the immediate postpartum period and the need for prompt acute care management.

The additional lessons to inform practice include the importance of a policy for the management of postpartum haemorrhage that is updated and rehearsed regularly via multidisciplinary 'drills'.

\section{A woman with a previous caesarean section, placenta percreta and subsequent postpartum haemorrhage}

A woman who had a previous caesarean section, presented at 23 weeks gestation with an antepartum haemorrhage (APH). She was diagnosed with placenta praevia. She had two further minor APHs and at 34 weeks gestation was admitted, contracting with small bright vaginal blood loss. She proceeded to an emergency caesarean section where a live baby was born. She died despite massive transfusions and multidisciplinary involvement. Autopsy showed placenta praevia percreta, with 
erosion into the posterior wall of the urinary bladder.

Source: Reproduced from Maternal deaths in Australia 2000-2002. Kildea S, Bennett M, Paech M, Section C: Cause of Maternal Death, page 34, 2006, with the permission of the National Perinatal Statistics Unit (10).

This vignette highlights the risks associated with placenta previa, particularly placenta percreta which adds significantly to the complexity in management.

There are a two unanswered questions from this vignette which potentially have great significance in the management of this case, that is: what level of facility was the woman booked in and what level of experience did the obstetrician performing the surgery possess? In their 2004 report (14), CEMACH identified the necessity to ensure that women known to be at a high risk of bleeding should be booked to give birth at a facility that would be able to deal with a massive haemorrhage, that is, access to blood banks; intensive care units and other specialist consultant care. In the same report they state that a consultant obstetrician should be in attendance at all caesareans for placenta praevia (14). A multidisciplinary approach including specialists, such as urologists, will also be useful in many cases.

An abnormally adherent placenta (placenta accreta, increta or percreta) is a rare complication of pregnancy that is predisposed to by prior uterine surgery, especially caesarean section. In the presence of a placenta praevia, the risk of placenta accreta increases dramatically depending on the number of caesareans performed, such that women with 3-4 prior caesarean sections and a placenta praevia have somewhere between a $50-70 \%$ risk of having a placenta accrete $(20,21)$. The key to avoiding a placenta accreta is to avoid the "first" caesarean section if possible.

This poses a great many challenges to clinicians. In their latest report (7) CEMACH make special mention regarding the identification and management particularly in those women who have had a previous caesarean section (as in this case) and have an anterior placenta. They recommend that "if there is any doubt magnetic resonance imaging (MRI) can be used along with ultrasound scanning in determining if the placenta is accreta or percreta” (page 78). In Australia this potentially poses issues regarding access to MRI and high definition ultrasound scanning in some areas.

This vignette presents a number of lessons that have resonance for maternity service planning and delivery. These include the need for women deemed to be at high risk of bleeding attending a hospital with suitable facilities to manage this potential event; having consultant obstetricians and anaesthetists attend caesarean sections for placenta praevia; and, ensuring that women are aware that caesarean section is not an entirely risk-free procedure and can hold problems for current and future pregnancies [3].

\section{A failure to recognise a woman's signs of intra-abdominal bleeding}

An African woman did not reveal her female genital mutilation (FGM) until late in pregnancy and was not referred to a specialist service for this. She had an elective caesarean section because it was considered that the consequences of her particular type of genital cutting would make vaginal delivery difficult. Although the operation was apparently straightforward, shortly afterwards she had a blood loss of several litres with a consequent fall in haemoglobin, which required transfusion. She continued to deteriorate with a steadily falling haemoglobin, rising pulse rate and 
falling blood pressure accompanied by poor urine output and increasing abdominal girth. Although she needed continual blood transfusion, an exploratory laparotomy was not performed until nearly two days later. By then she was in extremis, and several litres of blood were found in her abdomen. She sustained a cardiac arrest while still in theatre and could not be resuscitated.

Source: Reproduced from Saving Mothers' Lives: Reviewing maternal deaths to make motherhood safer - 2003-2005, Liston, W, Chapter 4: Haemorrhage, page 81, 2007, with the permission of the Confidential Enquiry into Maternal and Child Health (7).

This final vignette highlights the risks associated with elective caesarean section and the care of women with female genital mutilation (FGM). Although not obvious from the information in the report it is possible that there were language barriers and questions the appropriate use of interpreters. The lack of recognition of signs of significant blood loss, and therefore, appropriate management are concerning. CEMACH (7) recommended that "all staff require regular training on the identification and management of maternal collapse, including the identification of hidden bleeding and the management of haemorrhage" (page 19). This vignette highlights that elective caesarean sections are not without risk and women should be advised of this. The importance in having an agreed management plan for women with FGM; including antenatal discussion and intrapartum management was also highlighted as necessary.

\section{Implications for practice}

Whilst haemorrhage is often unpredictable there are a number of risk factors which can be identified. Clinicians should be aware of these risk factors in order to plan a woman's care with the appropriate consultation and referral to medical staff when required. The ACM's "National Midwifery Guidelines for Consultation and Referral" (22) provide a comprehensive framework for midwives to work within; there would be benefit in its adoption nationally. Table 3 lists the risk factors for postpartum haemorrhage and details the level of consultation and/or referral required of the midwife caring for the woman.

$<$ Table 3 here $>$

This article has identified a number of learning points for midwives, general practitioners and obstetricians, which complement the specific recommendations made by CEMACH (7) (page 78):

- All staff require regular training on identification and management of maternal collapse.

- An early warning scoring system may help in the more timely recognition of hidden bleeding.

- When severe haemorrhage occurs it is good practice to call straight away for the aid of colleagues with greater gynaecological surgical experience.

- The management of women with placenta percreta requires careful multidisciplinary planning in the antenatal period and the involvement of a consultant-led multidisciplinary team at birth.

- Guidelines for the management of women who refuse blood products must be made available to, and discussed with the woman and her family and all 
maternity staff as part of their routine training and continuing professional development.

- Women should be advised that caesarean section is not an entirely risk-free procedure and has potential problems in current and future pregnancies.

- All women who have had a previous caesarean section must have their placental site determined.

The challenge is for midwives, doctors and maternity services to incorporate these recommendations into everyday practice. Communication, collaboration and team work are crucial for success. Managers, clinicians and educators, both medical and midwifery, must work together to foster this team approach; multidisciplinary training can play an important role in achieving this aim.

Some Australian states have implemented specific programs to address some of these recommendations. For example, the NSW Department of Health have recently launched FONT (Fetal, Obstetric Emergency and Neonatal Resuscitation Training) mandatory training for all clinical staff working within Maternity Services in NSW (23). The FONT uses a multidisciplinary approach where medical and midwifery staff receive training together. King Edward Memorial Hospital for Women in Perth, Western Australia, run 6 weekly IN TIME (Interdisciplinary Management of Obstetric Emergencies) courses for all medical and midwifery staff. In addition to formalised training sessions, such as regular drills and in-service updates play an important role in developing and up-dating skills and competence. A focus needs to be on the recognition of maternal collapse and the appropriate management thereafter, the accurate estimation of maternal blood loss should be a priority.

Maternal blood loss is often underestimated which has implications for resuscitation efforts. Weighing pads and liners and measuring obvious blood loss are limited in their capacity to accurately determine blood loss. This underestimation has been shown to become more pronounced the larger the volume of blood lost (24). The importance of improving clinicians' skills at estimating blood loss cannot be stressed enough. When in doubt haemoglobin should be assessed. A particular challenge is in the immediate post-operative context where women are transferred from the operating theatre back to busy postnatal wards. Without the recognition that the woman has had a significant blood loss appropriate management and resuscitation cannot be commenced either immediately after the birth or later on a postnatal ward.

\section{Conclusion}

Maternal death is a rare event in Australia and other developed countries however when it occurs, the effects are traumatic for all involved. Midwives and maternity services have the opportunity, and a responsibility, to learn from these tragic events in order to reduce both mortality and morbidity for women in the future.

Obstetric haemorrhage is a leading cause of maternal mortality and the management of obstetric haemorrhage requires skilled, multidisciplinary care. All health care professionals caring for pregnant women should be skilled in the assessing the risk of intra and postpartum haemorrhage, in the initial management of haemorrhage and be prepared to call for expert help at the earliest opportunity. 
National maternal mortality reporting is an essential aspect of any effective health system and should be maintained at a high standard to ensure that learning can occur. Maternity care providers must understand the process for reporting maternal death in their country and ensure that they take the time to learn from the stories and recommendations. 


\section{References}


Table 1: Maternal deaths in Australia 2003-2005

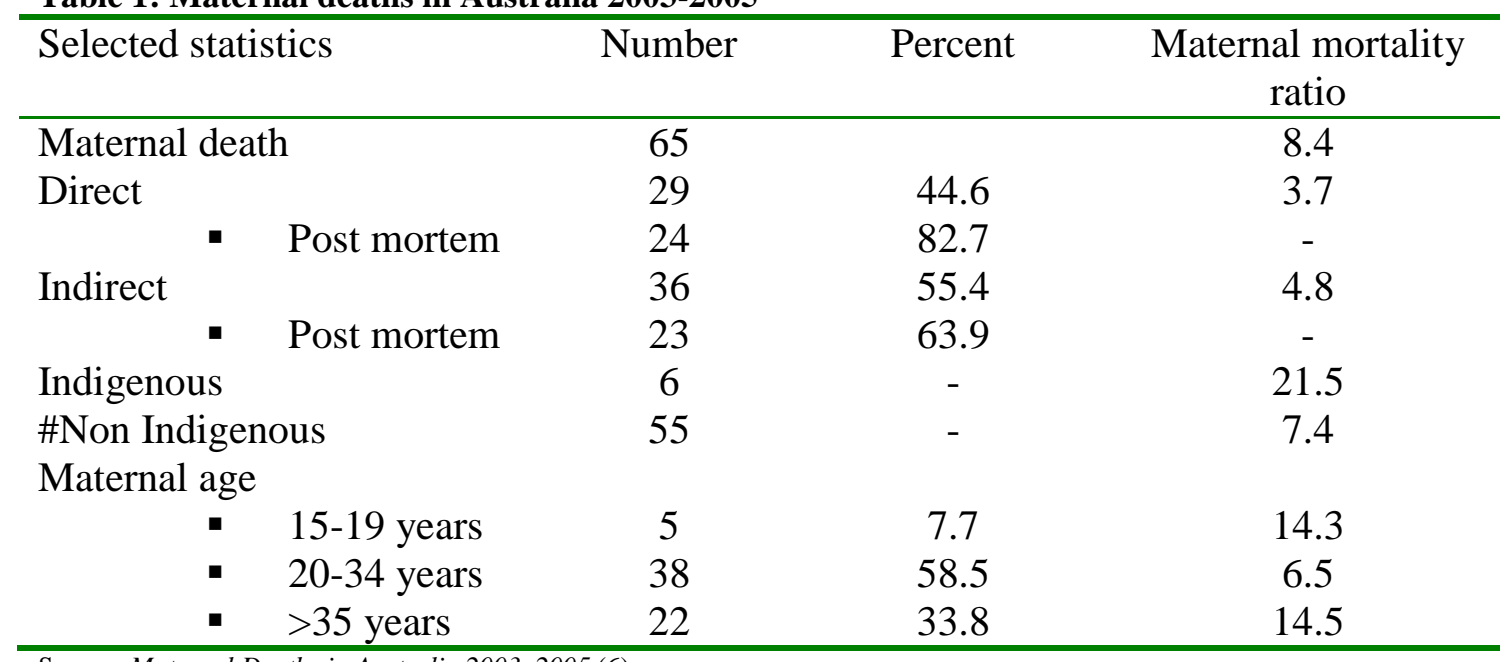

Source: Maternal Deaths in Australia 2003-2005 (6)

\#excludes deaths were Indigenous status not known from the non Indigenous numerator 
Table 2: Number and proportion of direct maternal deaths related to obstetric haemorrhage over total direct deaths: Australia and UK, 1994-2005

\begin{tabular}{lccccccc}
\hline \multicolumn{7}{c}{$\begin{array}{c}\text { Number and proportion of direct maternal deaths related to obstetric } \\
\text { haemorrhage over total direct deaths } \\
\text { Australia }\end{array}$} \\
& $\begin{array}{c}\text { Deaths from } \\
\text { Haemorrhage }\end{array}$ & Direct Deaths & $\%$ & $\begin{array}{c}\text { Deaths from } \\
\text { Haemorrhage }\end{array}$ & DK \\
$1994-96$ & $5^{\#}$ & 46 & 9 & $12^{\S}$ & 134 & 9 \\
$1997-99$ & $7 *$ & 34 & 21 & $7^{\S}$ & 106 & 7 \\
$2000-02$ & 9 & 32 & 28 & $17^{\S}$ & 106 & 16 \\
$2003-05$ & 4 & 29 & 14 & $14^{\S}$ & 132 & 11 \\
Total & $\mathbf{2 4}$ & $\mathbf{1 4 1}$ & $\mathbf{1 7}$ & $\mathbf{5 0}$ & $\mathbf{4 7 8}$ & $\mathbf{1 0}$ \\
\hline
\end{tabular}

\#There were 5 cases in which uteroplacental haemorrhage was the principal cause of death and 11 other cases in which uteroplacental haemorrhage was a contributory cause of death

*Defined as obstetric haemorrhage - Postpartum haemorrhage

§Includes placental abruption, placenta previa and postpartum haemorrhage 
Table 3: Risk factors for postpartum haemorrhage and the ACM's [19] recommended level of consultation and referral

Risk factors for postpartum haemorrhage

History of postpartum haemorrhage or retained placenta

High parity

Multiple pregnancy

Polyhydramnios

Fetal macrosomia

Anaemia

Placenta praevia, accreta or percreta

Placental abruption

Prolonged labour

Shoulder dystocia

Pre-eclampsia

Instrumental delivery

General anaesthesia

Fibroids

Mismanagement of the third stage

Retained placenta

Tocolytic drugs

Induced or augmented labours

Inversion of the uterus

Infection

Key: [19] A.

B. Consult with a medical practitioner.

C. Transfer responsibility for the woman's care to a medical specialist.

\section{Recommended level of consultation and referral}

$\mathrm{B} / \mathrm{C}$

$\mathrm{A} / \mathrm{B}$

$\mathrm{C}$

$\mathrm{B} / \mathrm{C}$

$\mathrm{B} / \mathrm{C}$

$\mathrm{B} / \mathrm{C}$

$\mathrm{C}$

C

B/C

C

C

C

C

B/C

C

C

C

C

C

C

another midwife and/or with a medical colleague.

1. World Health Organisation. International statistical classification of diseases and related health problems 10th revision (ICD-10) Vol II. Geneva: WHO; 1993.

2. United Nations. The Millennium Development Goals Report. New York: United Nations; 2008.

3. Sullivan E, King J, editors. Maternal deaths in Australia 2000-2002. Sydney: Australian Institute of Health and Welfare National Perinatal Statistics Unit; 2006.

4. WHO. Beyond the numbers, Reviewing maternal deaths and complications to make pregnancy safer. Geneva: World Health Organisation; 2002.

5. Kildea S, Pollock W, Barclay L. Making pregnancy safer in Australia: The importance of maternal death review. Australian \& New Zealand Journal of Obstetrics \& Gynaecology. 2008;48:130-6.

6. Sullivan E, Ball B, King J. Maternal Deaths in Australia 2002-2005. Sydney: Australian Institute for Health and Welfare; 2008.

7. Lewis G, editor. Saving Mothers' Lives: Reviewing maternal deaths to make motherhood safer - 2003-2005. London: Confidential Enquiry into Maternal and Child Health; 2007.

8. Lalonde A, Daviss B, ., Acosta A, Herschderfer K. Postpartum hemorrhage today: ICM/FIGO initiative 2004-2006. International Journal of Gynaecology \& Obstetrics. 2006 Sep;94(3):243-53. 
9. Geller S, Adams M, Kelly P, Kodkany B, Derman R. Postpartum hemorrhage in resource-poor settings. International Journal of Gynaecology \& Obstetrics. 2006 Mar;92(3):202-11.

10. Kildea S, Bennett M, Paech M. Haemorrhage. In: Sullivan E, King J, editors. Maternal deaths in Australia 2000-2002. Canberra: Australian Institute of Health and Welfare; 2006.

11. Liston W. Haemorrhage In: Lewis G, editor. The Confidential Enquiry into Maternal and Child Health (CEMACH) Saving Mothers' Lives: reviewing maternal deaths to make motherhood safer - 2003-2005 The Seventh Report on Confidential Enquiries into Maternal Deaths in the United Kingdom. London: CEMACH; 2007. p. 78-85.

12. Ford J, Sullivan E, Walters W, editors. Report on maternal deaths in Australia 1994-96. Canberra: National Health and Medical Research Council and Australian Institute of Health and Welfare National Perinatal Statistics Unit; 2001.

13. Slaytor E, Sullivan EA, King JF. Maternal Deaths in Australia 1997-1999.

Canberra: Australian Institute of Health and Welfare and National Perinatal Statistics Unit; 2004.

14. Lewis G, editor. Why mothers die, The sixth report of the confidential enquiries into maternal deaths in the United Kingdom, 2000-2002. London: RCOG Press; 2004.

15. Lewis G, Drife J, editors. Why mothers die 1997-1999, The fifth report of the confidential enquiries into maternal deaths in the United Kingdom. London: RCOG Press; 2001.

16. Joseph KS, Rouleau J, Kramer MS, Young DC, Liston RM, Baskett TF. Investigation of an increase in postpartum haemorrhage in Canada. BJOG: An International Journal of Obstetrics and Gynaecology. 2007;114(6):751-9.

17. Ford J, Roberts C, Bell J, Algert C, Morris J. Postpartum haemorrhage occurrence and recurrence: a population-based study. Medical Journal of Australia. 2007

187(7):391-3.

18. Ford J, Roberts C, Simpson J, Vaughan J, Cameron C. Increased postpartum hemorrhage rates in Australia. International Journal of Gynecology \& Obstetrics. 2007;98(3):237-43.

19. Cameron CA, Roberts CL, Olive EC, Ford JB, Fischer WE. Trends in postpartum haemorrhage. Australian \& New Zealand Journal of Public Health. 2006 Apr;30(2):151-6.

20. Silver R, Landon M, Rouse D, Leveno K, Spong C, Thom E, et al. Maternal morbidity associated with multiple repeat cesarean deliveries. Obsterics and Gynecology. 2006;107(6):1226-32.

21. Yang Q, Wen S, Oppenheimer L, Chen X, Black D, Gao J, et al. Association of caesarean delivery for first birth with placenta praevia and placental abruption in second pregnancy. BJOG. 2007;114:609-13.

22. ACMI. National Midwifery Guidelines for Consultation and Referral. Canberra: Australian College of Midwives Inc.; 2004.

23. NSW Department of Health. Information Bulletin: Maternity Education: Fetal Welfare Assessment, Maternity Emergency Management and Neonatal Resuscitation Training. Sydney NSW Health; 2007.

24. Buckland S, Homer C. Estimating blood loss after birth: using simulated clinical examples. Women and Birth. 2007;20(2):85-8. 